Pacific Journal of Mathematics

OPERATORS SIMILAR TO UNITARY OR SELFADJOINT ONES 


\title{
OPERATORS SIMILAR TO UNITARY OR SELFADJOINT ONES
}

\author{
JAN A. VAN CASTEREN
}

\begin{abstract}
Let $T$ be a bounded linear operator on a Hilbert space. General necessary and sufficient conditions are given in order that $V T V^{-1}$ is unitary for some bounded linear operator $V$ with bounded everywhere defined inverse. Similarly let $B$ be a closed and densely defined linear operator in a Hilbert space. General necessary and sufficient conditions are given in order that $V B V^{-1}$ is selfadjoint for some bounded linear operator $V$ with bounded everywhere defined inverse.
\end{abstract}

1. Introduction and some preliminaries. Throughout this paper $\mathbf{H}$ is a complex Hilbert space with inner-product (, ). Let $A$ and $B$ be linear operators with domain and range in $\mathbf{H}$. Then $A$ and $B$ are said to be similar if there exists a continuous linear operator $V: \mathbf{H} \rightarrow \mathbf{H}$ with bounded everywhere defined inverse such that $V A=B V$. Let $T: \quad \mathbf{H} \rightarrow \mathbf{H}$ be a bounded linear operator. Then $T$ is said to be power bounded if $\sup \left\{\left\|T^{n}\right\|: n \in \mathbf{N}\right\}$ is finite. Again let $T: \mathbf{H} \rightarrow \mathbf{H}$ be a continuous linear operator and suppose that its spectrum is contained in the circumference of the closed unit disc. The problem which poses itself is to find conditions on the resolvent family $\left\{(\lambda I-T)^{-1}:|\lambda| \neq 1\right\}$ which guarantee that $T$ is similar to a unitary operator. Next let $A$ be a closed linear operator with domain and range in $\mathbf{H}$. Suppose that its spectrum is a subset of $\mathbf{R}$. Find necessary and sufficient conditions on the resolvent family $\left\{(\lambda I-i A)^{-1}: \operatorname{Re} \lambda \neq 0\right\}$ in order that $A$ is similar to a selfadjoint operator. The main tool we use is what might be called an operator valued Poisson kernel. If the spectrum of $T$ is a subset of $\{\lambda \in \mathbf{C}:|\lambda|=1\}$ and if $T$ has inverse $S$, the corresponding Poisson kernel is given by

$$
\left(1-r^{2}\right)\left(I-r e^{-i \theta} T\right)^{-1}\left(I-r e^{i \theta} S\right)^{-1}, \quad 0 \leq r \leq 1,-\pi \leq \theta \leq+\pi .
$$

If $A$ is a closed linear operator in $\mathbf{H}$ the spectrum of which is a subset of $\mathbf{R}$, then the corresponding Poisson kernel is given by

$$
\begin{aligned}
\omega\left(\omega^{2} I+\right. & \left.(\xi I-A)^{2}\right)^{-1} \\
& =\omega\left(((\omega+i \xi) I-i A)^{-1}((\omega-i \xi) I+i A)^{-1}\right), \quad \omega>0, \xi \in \mathbf{R} .
\end{aligned}
$$

The present results generalize Theorems 1 and 2 in Van Casteren [9], where more related references can be found too. They are also closely related to a problem posed by Sz.-Nagy in [3, p. 585]. See also Sz.-Nagy and Foiaş [8, Chapitre IX, p. 334]. Another closely related paper is Stampfli [5]. This reference should also have been given in [9]. 
First we shall prove some lemmas which will be useful in the sequel.

1.1. LeMMA (a) Let $\left(\alpha_{k}: k \in \mathbf{N}\right)$ be a sequence of nonnegative real numbers. The following inequalities hold:

$$
\begin{aligned}
e^{-1} \sup \left\{(n+1)^{-1} \sum_{k=0}^{n} \alpha_{k}: n \in \mathbf{N}\right\} & \leq \sup \left\{(1-r) \sum_{k=0}^{\infty} r^{k} \alpha_{k}: 0 \leq r<1\right\} \\
& \leq \sup \left\{(n+1)^{-1} \sum_{k=0}^{n} \alpha_{k}: n \in \mathbf{N}\right\} .
\end{aligned}
$$

(b) Let $\alpha:[0, \infty) \rightarrow[0, \infty)$ be a Borel measurable function. Then

$$
\begin{aligned}
e^{-1} \sup \left\{t^{-1} \int_{0}^{t} \alpha(s) d s: t>0\right\} & \leq \sup \left\{\omega \int_{0}^{\infty} e^{-\omega s} \alpha(s) d s: \omega>0\right\} \\
& \leq \sup \left\{t^{-1} \int_{0}^{t} \alpha(s) d s: t>0\right\}
\end{aligned}
$$

Proof. (a) The following (in-) equalities are readily verified:

$$
\begin{aligned}
(1-r) r^{n} \sum_{k=0}^{n} \alpha_{k} & \leq(1-r) \sum_{k=0}^{\infty} r^{k} \alpha_{k}=(1-r)^{2} \sum_{k=0}^{\infty} r^{k} \sum_{j=0}^{k} \alpha_{j} \\
& \leq(1-r)^{2}\left(\sup _{k \in \mathbf{N}}(k+1)^{-1} \sum_{j=0}^{k} \alpha_{j}\right) \sum_{k=0}^{\infty}(k+1) r^{k} \\
& =\sup _{k \in \mathbf{N}}(k+1)^{-1} \sum_{j=0}^{k} \alpha_{j}, \quad 0 \leq r<1, n \in \mathbf{N} .
\end{aligned}
$$

From this (a) follows with $r=n(n+1)^{-1}$.

(b) As in the proof of (a) we have

$$
e^{-1} \omega \int_{0}^{\omega^{-1}} \alpha(s) d s \leq \omega \int_{0}^{\infty} e^{-\omega s} \alpha(s) d s=\omega^{2} \int_{0}^{\infty} e^{-\omega t}\left(\int_{0}^{t} \alpha(s) d s\right) d t
$$

The following lemma will be needed for the proof of Theorem 3.1.

1.2. LEMMA. Let $h$ be a complex valued harmonic function on the right half plane for which

$$
M:=\sup \left\{\int_{-\infty}^{\infty}|h(\omega, \xi)| d \xi: \omega>0\right\}
$$

is finite. The following assertions hold true. 
(a) The function $h$ satisfies the following inequality:

$$
3 \pi \omega|h(\omega, \xi)| \leq 4 M, \quad \omega>0, \xi \in \mathbf{R} .
$$

(b) There exists a complex measure $\mu$ on $\mathbf{R}$, which is of bounded total variation, such that

$$
h(\omega, \xi)=\frac{\omega}{\pi} \int_{-\infty}^{\infty} \frac{1}{\omega^{2}+(\xi-\eta)^{2}} d \mu(\eta), \quad \omega>0, \xi \in \mathbf{R} .
$$

(c) Suppose that $h$ is of the form

$$
h(\omega, \xi)=F(\omega+i \xi)+G(\omega-i \xi), \quad \omega>0, \xi \in \mathbf{R},
$$

where $F$ and $G$ are holomorphic and where

$$
\sup \{\omega|F(\omega)|: \omega>0\}
$$

is finite. Then

$$
F(\lambda)=(2 \pi)^{-1} \int_{-\infty}^{\infty}(\lambda-i \eta)^{-1} d \mu(\eta), \quad \operatorname{Re} \lambda>0
$$

and

$$
G(\lambda)=(2 \pi)^{-1} \int_{-\infty}^{\infty}(\lambda+i \eta)^{-1} d \mu(\eta), \quad \operatorname{Re} \lambda>0
$$

Proof. (a) The reader is referred to Duren [1, Lemma 1, p. 188].

(b) We refer the reader to Stein [6, Theorem 2, Corollary, p. 200].

(c) Let $\lambda$ in $C$ be such that $\operatorname{Re} \lambda>0$. Then

$$
\begin{array}{rl}
F(\lambda)-(2 \pi)^{-1} \int_{-\infty}^{\infty}(\lambda-i \eta)^{-1} & d \mu(\eta) \\
& =(2 \pi)^{-1} \int_{-\infty}^{\infty}(\bar{\lambda}+i \eta)^{-1} d \mu(\eta)-G(\bar{\lambda}) .
\end{array}
$$

Since the left-hand side of this equality is analytic in $\lambda$ and since the right-hand side is analytic in $\bar{\lambda}$, it follows that each side is constant. Since $\sup \{\omega|F(\omega)|: \omega>0\}$ is finite, we conclude that this constant is zero.

Remark. Let $h$ be as in Lemma 1.2. Fix $\omega^{\prime}>0$. By (a) the harmonic function

$$
(\omega, \xi) \mapsto h\left(\omega+\omega^{\prime}, \xi\right)-\frac{\omega}{\pi} \int_{-\infty}^{\infty} \frac{1}{\omega^{2}+(\xi-\eta)^{2}} h\left(\omega^{\prime}, \eta\right) d \eta,
$$

$$
\omega>0, \xi \in \mathbf{R},
$$


is bounded. Since it has boundary value 0 , it vanishes identically. So

$$
h(\omega, \xi)=\lim _{\omega^{\prime} \downarrow 0} h\left(\omega+\omega^{\prime}, \xi\right)=\lim _{\omega^{\prime} \downarrow 0} \frac{\omega}{\pi} \int_{-\infty}^{\infty} \frac{1}{\omega^{2}+(\xi-\eta)^{2}} h\left(\omega^{\prime}, \eta\right) d \eta .
$$

An application of the Riesz representation theorem for $C_{0}(\mathbf{R})$ (e.g. see Hewitt and Ross [4, Theorem 14.4, p. 168]) and of the fact that the dual unit ball of $C_{0}(\mathbf{R})$ is weak* compact, yields the existence of a measure $\mu$ for which (b) holds.

2. Operators which are similar to a unitary one. In this section $\mathbf{H}$ is a complex Hilbert space and $T: \mathbf{H} \rightarrow \mathbf{H}$ is a continuous linear operator with a bounded everywhere defined inverse $S$. The theorem we want to prove reads as follows. Notice that the equivalence of (i) and (iii) yields a positive answer to Question 1 in Stampfli [5, p. 149].

2.4. THEOREM. The following assertions are equivalent:

(i) $T$ is similar to a unitary operator;

(ii) $T$ and $S$ are power bounded;

(iii) $T$ is power bounded, $(I-\lambda S)^{-1}$ exists for $|\lambda|<1$ and

$$
\sup \left\{(1-|\lambda|)\left\|(I-\lambda S)^{-1}\right\|:|\lambda|<1\right\}
$$

is finite;

(iv) For each $x$ in $\mathbf{H}$ the expressions

$$
\sup \left\{(n+1)^{-1} \sum_{k=0}^{n}\left\|T^{k} x\right\|^{2}: n \in \mathbf{N}\right\}
$$

and

$$
\sup \left\{(n+1)^{-1} \sum_{k=0}^{n}\left\|\left(T^{*}\right)^{k} x\right\|^{2}: n \in \mathbf{N}\right\}
$$

are finite, $(I-\lambda S)^{-1}$ exists for $|\lambda|<1$ and

$$
\sup \left\{(1-|\lambda|)\left\|(I-\lambda S)^{-1}\right\|:|\lambda|<1\right\}
$$

is finite;

(v) For every $x$ in $\mathbf{H}$ the expressions

$$
\sup \left\{(n+1)^{-1} \sum_{k=0}^{n}\left\|\left(T^{*}\right)^{k} x\right\|^{2}: n \in \mathbf{N}\right\}
$$


and

$$
\sup \left\{(n+1)^{-1} \sum_{k=0}^{n}\left\|S^{k} x\right\|^{2}: n \in \mathbf{N}\right\}
$$

are finite;

(vi) For $|\lambda|<1$ the inverses $(I-\lambda T)^{-1}$ and $(I-\lambda S)^{-1}$ exist and for every $x$ and $y$ in $\mathbf{H}$ the expression

$$
\sup \left\{\left(1-r^{2}\right) \int_{-\pi}^{+\pi}\left|\left(\left(I-r e^{-i \theta} T\right)^{-1}\left(I-r e^{i \theta} S\right)^{-1} x, y\right)\right| d \theta: 0 \leq r<1\right\}
$$

is finite.

The proof of the equivalency of (ii) and (iii) is contained in Van Casteren [8, Theorem 1].

Proof. The proof of the equivalency of (i) and (ii) appears in Sz.-Nagy [7]. The implication (ii) $\Rightarrow$ (iii) is easy. The implication (iii) $\Rightarrow$ (iv) is trivial.

(iv) $\Rightarrow$ (vi) Put

$$
M=\sup \left\{\left\|\left\{\left(1-|\lambda|^{2}\right)(I-\lambda S)^{-1}-I\right\} T\right\||\lambda|^{-1}: 0<|\lambda|<1\right\} .
$$

From (iv) it follows that $M$ is finite. Next fix $x$ and $y$ in $\mathbf{H}$ and put

$$
M_{1}(x)^{2}=\sup \left\{\left(1-r^{2}\right) \sum_{k=0}^{\infty} r^{2 k}\left\|T^{k} x\right\|^{2}: 0 \leq r<1\right\}
$$

and

$$
M_{2}(y)^{2}=\sup \left\{\left(1-r^{2}\right) \sum_{k=0}^{\infty} r^{2 k}\left\|\left(T^{*}\right)^{k} y\right\|^{2}: 0 \leq r<1\right\} .
$$

From Lemma 1.1 and assertion (iv) it follows that $M_{1}(x)$ and $M_{2}(y)$ are finite. For $0<|\lambda|<1$ we have

$$
\begin{aligned}
& \left((I-\bar{\lambda} T)^{-1}(I-\lambda S)^{-1} x, y\right) \\
& \quad=\left(\lambda^{-1}\left\{\left(1-|\lambda|^{2}\right)(I-\lambda S)^{-1}-I\right\} T(I-\bar{\lambda} T)^{-1} x,\left(I-\lambda T^{*}\right)^{-1} y\right) .
\end{aligned}
$$

Hence

$$
\left|\left((I-\bar{\lambda} T)^{-1}(I-\lambda S)^{-1} x, y\right)\right| \leq M\left\|(I-\bar{\lambda} T)^{-1} x\right\|\left\|\left(I-\lambda T^{*}\right)^{-1} y\right\| .
$$


So

$$
\begin{aligned}
& \int_{-\pi}^{+\pi}\left|\left(\left(I-r e^{-i \theta} T\right)^{-1}\left(I-r e^{i \theta} S\right)^{-1} x, y\right)\right| d \theta \\
& \quad \leq M \int_{-\pi}^{+\pi}\left\|\left(I-r e^{-i \theta} T\right)^{-1} x\right\|\left\|\left(I-r e^{i \theta} T^{*}\right)^{-1} y\right\| d \theta \\
& \quad \leq M\left(\int_{-\pi}^{+\pi}\left\|\left(I-r e^{-i \theta} T\right)^{-1} x\right\|^{2} d \theta\right)^{1 / 2}\left(\int_{-\pi}^{+\pi}\left\|\left(I-r e^{i \theta} T^{*}\right)^{-1} y\right\|^{2} d \theta\right)^{1 / 2} \\
& \quad=2 \pi M\left(\sum_{k=0}^{\infty} r^{2 k}\left\|T^{k} x\right\|^{2}\right)^{1 / 2}\left(\sum_{k=0}^{\infty} r^{2 k}\left\|\left(T^{*}\right)^{k} y\right\|^{2}\right)^{1 / 2} \\
& \quad \leq 2 \pi M M_{1}(x) M_{2}(y)\left(1-r^{2}\right)^{-1} .
\end{aligned}
$$

Hence (vi) follows.

(vi) $\Rightarrow$ (ii) Fix $x$ and $y$ in $\mathbf{H}$. Since $T=S^{-1}$ it follows that

$$
\begin{aligned}
\left(1-r^{2}\right)\left(I-r e^{-i \theta} T\right)^{-1}\left(I-r e^{i \theta} S\right)^{-1} & \\
= & \sum_{k=-\infty}^{\infty} r^{|k|} e^{i k \theta} S^{k}, \quad 0 \leq r<1,-\pi \leq \theta \leq+\pi .
\end{aligned}
$$

So

$$
\begin{aligned}
& r^{|n|}\left(T^{n} x, y\right) \\
& \quad=\frac{1-r^{2}}{2 \pi} \int_{-\pi}^{+\pi} e^{i n \theta}\left(\left(I-r e^{-i \theta} T\right)^{-1}\left(I-r e^{i \theta} S\right)^{-1} x, y\right) d \theta, \quad n \in \mathbf{Z} .
\end{aligned}
$$

With $r=|n|(|n|+1)^{-1}$ we infer

$$
\left|\left(T^{n} x, y\right)\right| \leq e \sup _{0 \leq r<1} \frac{1-r^{2}}{2 \pi} \int_{-\pi}^{+\pi}\left|\left(\left(I-r e^{-\imath \theta} T\right)^{-1}\left(I-r e^{\imath \theta} S\right)^{-1} x, y\right)\right| d \theta .
$$

So $\sup \left\{\left|\left(T^{n} x, y\right)\right|: n \in \mathbf{Z}\right\}$ is finite for each $x$ and $y$ in $\mathbf{H}$. By a Banach Steinhaus argument it follows that $\sup \left\{\left\|T^{n}\right\|: n \in \mathbf{Z}\right\}$ is finite. Hence (ii) follows.

(ii) $\Rightarrow$ (v) Trivial.

(v) $\Rightarrow$ (vi) Fix $x$ and $y$ in H. Put

$$
M_{1}(x)^{2}=\sup \left\{\left(1-r^{2}\right) \sum_{k=0}^{\infty} r^{2 k}\left\|S^{k} x\right\|^{2}: 0 \leq r<1\right\}
$$

and put

$$
M_{2}(y)^{2}=\sup \left\{\left(1-r^{2}\right) \sum_{k=0}^{\infty} r^{2 k}\left\|\left(T^{*}\right)^{k} y\right\|^{2}: 0 \leq r<1\right\} .
$$


From Lemma 1.1 and (v) it follows that $M_{1}(x)$ and $M_{2}(y)$ are finite. For $0 \leq r<1$ we have

$$
\begin{aligned}
\left(1-r^{2}\right) \int_{-\pi}^{+\pi} \mid((I & \left.\left.-r e^{-i \theta} T\right)^{-1}\left(-r e^{i \theta} S\right)^{-1} x, y\right) \mid d \theta \\
= & \left(1-r^{2}\right) \int_{-\pi}^{+\pi}\left|\left(\left(I-r e^{i \theta} S\right)^{-1} x,\left(I-r e^{i \theta} T^{*}\right)^{-1} y\right)\right| d \theta \\
\leq & \left(1-r^{2}\right)\left(\int_{-\pi}^{+\pi}\left\|\left(I-r e^{i \theta} S\right)^{-1} x\right\|^{2} d \theta\right)^{1 / 2} \\
& \times\left(\int_{-\pi}^{+\pi}\left\|\left(I-r e^{i \theta} T^{*}\right)^{-1} y\right\|^{2} d \theta\right)^{1 / 2} \leq 2 \pi M_{1}(x) M_{2}(y) .
\end{aligned}
$$

Hence (vi) follows.

Next we shall give an example of an operator $T: l^{2}(\mathbf{Z}) \rightarrow l^{2}(\mathbf{Z})$ which is not similar to a unitary operator but for which, for each $x$ in $l^{2}(\mathbf{Z})$, the expression

$$
\sup \left\{(2 n+1)^{-1} \sum_{j=-n}^{+n}\left\|T^{J} x\right\|^{2}: n \in \mathbf{N}\right\}
$$

is finite.

EXAMPLE 1. Fix $0<2 \gamma<1$ and put $\alpha_{k}=(1+|k|)^{-\gamma}, k \in \mathbf{Z}$. Let $\left(e_{k}: k \in \mathbf{Z}\right)$ be the standard basis in $l^{2}(\mathbf{Z})$ and define the operator $T: l^{2}(\mathbf{Z}) \rightarrow l^{2}(\mathbf{Z})$ by

$$
T e_{k}=\frac{\alpha_{k+1}}{\alpha_{k}} e_{k+1}, \quad k \in \mathbf{Z}
$$

Then

$$
T^{j} e_{k}=\frac{\boldsymbol{\alpha}_{k+j}}{\boldsymbol{\alpha}_{k}} e_{k+j}, \quad k, j \in \mathbf{Z}
$$

Fix $x$ in $l^{2}(\mathbf{Z})$. Then

$$
\sum_{j=-n}^{+n}\left\|T^{j} x\right\|^{2} \leq\|x\|^{2} . \sup _{k \in \mathbf{Z}} \alpha_{k}^{-2} \sum_{j=-n}^{+n} \alpha_{k+j}^{2}
$$

Since $0<2 \gamma<1$, it follows that

$$
k \in \sup _{\mathbf{z}, n \in \mathbf{N}} \frac{1}{(2 n+1) \alpha_{k}^{2}} \sum_{j=-n}^{+n} \alpha_{k+j}^{2}
$$


is finite. Consequently

$$
\sup \left\{(2 n+1)^{-1} \sum_{j=-n}^{+n}\left\|T^{j} x\right\|^{2}: n \in \mathbf{N}\right\}
$$

is finite. Since $\left\|T^{J}\right\|=(1+|j|)^{\gamma}$, it follows that $T$ cannot be similar to a unitary operator.

3. Operators which are similar to a selfadjoint one. In this section $A$ denotes a linear operator with domain and range in a Hilbert space $\mathbf{H}$. The operator $i A$ is said to generate a strongly continuous semigroup $\left\{P_{t}: t \geq 0\right\}$ if

$$
i A=\mathrm{s}-\lim _{t \downarrow 0} \frac{P_{t}-I}{t}
$$

It generates a strongly continuous group $\left\{P_{t}: t \in \mathbf{R}\right\}$ if

$$
i A=\mathrm{s}-\lim _{t \rightarrow 0} \frac{P_{t}-I}{t} .
$$

For more details on semigroups see Yosida [10, Chapter IX]. For the proof of Theorem 3.1. we need Stone's representation theorem; see Yosida [10, Corollary 2, p. 253]. Furthermore we shall use Plancherel's theorem in $L^{2}(\mathbf{R}, \mathbf{H})$; e.g. see Edwards and Gaudry $[2, \S 3.4$, p. 53] or Stein [6, Chapter II, §5, pp. 45-47].

We want to prove the following theorem

3.1. TheOREM. Let $A$ be a linear operator with domain and range in a Hilbert space $\mathbf{H}$. The following assertions are equivalent.

(i) The operator $A$ is similar to a selfadjoint operator;

(ii) The operator iA generates a bounded strongly continuous group;

(iii) The operator -iA generates a bounded strongly continuous semigroup, $(\lambda I-i A)^{-1}$ exists for $\operatorname{Re} \lambda>0$ and

$$
\sup \left\{\operatorname{Re} \lambda\left\|(\lambda I-i A)^{-1}\right\|: \operatorname{Re} \lambda>0\right\}
$$

is finite;

(iv) The operator -iA generates a strongly continuous semi-group $\left\{P_{t}: t \geq 0\right\}$ for which the expressions

$$
\sup \left\{t^{-1} \int_{0}^{t}\left\|P_{s} x\right\|^{2} d s: t>0\right\} \quad \text { and } \sup \left\{t^{-1} \int_{0}^{t}\left\|P_{s}^{*} x\right\|^{2} d s: t>0\right\}
$$


are finite for each $x$ in $\mathbf{H}$, for which $(\lambda I-i A)^{-1}$ exists for $\operatorname{Re} \lambda>0$ and for which

$$
\sup \left\{\operatorname{Re} \lambda\left\|(\lambda I-i A)^{-1}\right\|: \operatorname{Re} \lambda>0\right\}
$$

is finite;

(v) The operator -iA generates a strongly continuous group $\left\{P_{t}: t \in \mathbf{R}\right\}$ for which the expressions

$$
\sup \left\{t^{-1} \int_{0}^{t}\left\|P_{s}^{*} x\right\|^{2} d s: t>0\right\} \quad \text { and } \sup \left\{t^{-1} \int_{0}^{t}\left\|P_{-s} x\right\|^{2} d s: t>0\right\}
$$

are finite for each $x$ in $\mathbf{H}$;

(vi) The operator $A$ is closed, $(\lambda I-i A)^{-1}$ exist for $\operatorname{Re} \lambda \neq 0$ and for each $x, y$ in $\mathbf{H}$ the expression

$$
\sup \left\{2 \omega \int_{-\infty}^{\infty}\left|\left(\left(\omega^{2} I+(\xi I-A)^{2}\right)^{-1} x, y\right)\right| d \xi: \omega>0\right\}
$$

is finite and

$$
\lim _{\lambda \rightarrow \infty} \lambda\left((\lambda I+i A)^{-1} x, y\right)=(x, y)
$$

Proof. The equivalency of (i) and (ii) follows from Stone's Theorem (see Yosida 1.c.) and from Sz.-Nagy [7].

(ii) $\Rightarrow$ (iii) Let $-i A$ be the generator of the bounded group $\left\{P_{t}: t \in \mathbf{R}\right\}$. For $\operatorname{Re} \lambda>0$ we have

$$
(\lambda I-i A)^{-1} x=\int_{0}^{\infty} e^{-\lambda s} P_{-s} x d s, \quad x \in \mathbf{H} .
$$

Put $M=\sup \left\{\left\|P_{-s}\right\|: s \geq 0\right\}$. Then

$$
\operatorname{Re} \lambda\left\|(\lambda I-i A)^{-1}\right\| \leq M, \quad \operatorname{Re} \lambda>0 .
$$

(iii) $\Rightarrow$ (iv) Trivial.

(iv) $\Rightarrow$ (vi) Let $-i A$ and $\left\{P_{t}: t \geq 0\right\}$ be as in (iv). Put

$$
M_{1}(x)^{2}=\sup \left\{t^{-1} \int_{0}^{t}\left\|P_{s} x\right\|^{2} d s: t>0\right\}, \quad x \in \mathbf{H}
$$

put

$$
M_{2}(x)^{2}=\sup \left\{t^{-1} \int_{0}^{t}\left\|P_{s}^{*} x\right\|^{2} d s: t>0\right\}, \quad x \in \mathbf{H}
$$


and put

$$
M=\sup \left\{\left\|2 \operatorname{Re} \lambda(\lambda I-i A)^{-1}-I\right\|: \operatorname{Re} \lambda>0\right\} .
$$

Let $\lambda=\omega+i \xi$ in $\mathbf{C}$ be such that $\omega=\operatorname{Re} \lambda>0$ and let $x$ and $y$ be in $\mathbf{H}$. Then

$$
\begin{aligned}
\left(\left(\omega^{2} I+\right.\right. & \left.\left.(\xi I-A)^{2}\right)^{-1} x, y\right) \\
& =\left(\left\{2 \omega(\lambda I-i A)^{-1}-I\right\}(\bar{\lambda} I+i A)^{-1} x,\left(\lambda I-i A^{*}\right)^{-1} y\right) .
\end{aligned}
$$

From (3), the definition of $M$, it follows that

$$
\left|\left(\left(\omega^{2} I+(\xi I-A)^{2}\right)^{-1} x, y\right)\right| \leq M\left\|(\bar{\lambda} I+i A)^{-1} x\right\|\left\|\left(\lambda I-i A^{*}\right)^{-1} y\right\| .
$$

So by Schwarz' inequality we infer

$$
\begin{aligned}
\left(\int_{-\infty}^{\infty} \mid\left(\left(\omega^{2} I+\right.\right.\right. & \left.\left.\left.(\xi I-A)^{2}\right)^{-1} x, y\right) \mid d \xi\right)^{2} \\
\leq & M^{2} \int_{-\infty}^{\infty}\left\|((\omega-i \xi) I+i A)^{-1} x\right\|^{2} d \xi \\
& \cdot \int_{-\infty}^{\infty}\left\|\left((\omega+i \xi) I-i A^{*}\right)^{-1} y\right\|^{2} d \xi
\end{aligned}
$$

Since $-i A$ generates the semigroup $\left\{P_{t}: t \geq 0\right\}$, the operator $+i A^{*}$ generates the semigroup $\left\{P_{t}^{*}: t \geq 0\right\}$; see Yosida [10, Chapter VII, Theorem 3, p. 196 and Chapter IX, §13]. Since

$$
((\omega-i \xi) I+i A)^{-1} x=\int_{0}^{\infty} e^{-(\omega-i \xi) s} P_{s} x d s
$$

and since

$$
\left((\omega+i \xi) I-i A^{*}\right)^{-1} x=\int_{0}^{\infty} e^{-(\omega+i \xi) s} P_{s}^{*} x d s
$$

it follows form (4) and from Plancherel's theorem in Hilbert space that

$$
\begin{aligned}
& \left(\frac{1}{2 \pi} \int_{-\infty}^{\infty}\left|\left(\left(\omega^{2} I+(\xi I-A)^{2}\right)^{-1} x, y\right)\right| d \xi\right)^{2} \\
& \quad \leq M^{2} \int_{0}^{\infty} e^{-2 \omega s}\left\|P_{s} x\right\|^{2} d s \int_{0}^{\infty} e^{-2 \omega s}\left\|P_{s}^{*} y\right\|^{2} d s
\end{aligned}
$$

From Lemma 1.1(b) and by (1) and (2) it follows that

$$
2 \omega \int_{-\infty}^{\infty}\left|\left(\left(\omega^{2} I+(\xi I-A)^{2}\right)^{-1} x, y\right)\right| d \xi \leq 2 \pi M M_{1}(x) M_{2}(y) .
$$

Since $M, M_{1}(x)$ and $M_{2}(y)$ are finite, assertion (vi) follows. 
(vi) $\Rightarrow$ (ii) Fix $x$ and $y$ in $\mathbf{H}$. The function

$$
(\omega, \xi) \mapsto 2 \omega\left(\left(\omega^{2} I+(\xi I-A)^{2}\right)^{-1} x, y\right), \quad \omega>0, \xi \in \mathbf{R},
$$

is harmonic and by (vi)

$$
\sup \left\{2 \omega \int_{-\infty}^{\infty}\left|\left(\left(\omega^{2} I+(\xi I-A)^{2}\right)^{-1} x, y\right)\right| d \xi: \omega>0\right\}
$$

is finite. So by Lemma 1.2(a) there exists a complex Borel measure $\mu_{x, y}$, which is of bounded total variation, such that

$$
\begin{aligned}
2 \omega\left(\left(\omega^{2} I\right.\right. & \left.\left.+(\xi I-A)^{2}\right)^{-1} x, y\right) \\
& =\frac{\omega}{\pi} \int_{-\infty}^{\infty}\left(\omega^{2}+(\xi-\eta)^{2}\right)^{-1} d \mu_{x, y}(\eta), \quad \omega>0, \xi \in \mathbf{R} .
\end{aligned}
$$

Since $\lim _{\lambda \rightarrow \infty} \lambda\left((\lambda I+i A)^{-1} x, y\right)=(x, y)$ it follows from Lemma $1.2(\mathrm{c})$ that

(5) $\quad\left((\lambda I-i A)^{-1} x, y\right)=(2 \pi)^{-1} \int_{-\infty}^{\infty}(\lambda-i \eta)^{-1} d \mu_{x, y}(\eta), \quad \operatorname{Re} \lambda>0$

It also follows that

(6) $\left((\lambda I+i A)^{-1} x, y\right)=(2 \pi)^{-1} \int_{-\infty}^{\infty}(\lambda+i \eta)^{-1} d \mu_{x, y}(\eta), \quad \operatorname{Re} \lambda>0$

We also conclude that

$$
2 \pi(x, y)=\mu_{x, y}(\mathbf{R})
$$

Next consider, for $\omega>0$ and $t$ in $\mathbf{R}$, the identities

$$
\begin{aligned}
\int_{-\infty}^{\infty} e^{\omega|t|+i \xi t} \cdot 2 \omega\left(\left(\omega^{2} I+(\xi I-A)^{2}\right)^{-1} x, y\right) d \xi \\
=\int_{-\infty}^{\infty} e^{\omega|t|+i \xi t}\left(\frac{\omega}{\pi} \int_{-\infty}^{\infty}\left(\omega^{2}+(\xi-\eta)^{2}\right)^{-1} d \mu_{x, y}(\eta)\right) d \xi \\
=\int_{-\infty}^{\infty} e^{i \eta t} e^{\omega|t|}\left(\frac{\omega}{\pi} \int_{-\infty}^{\infty}\left(\omega^{2}+(\xi-\eta)^{2}\right)^{-1} e^{i(\xi-\eta) t} d \xi\right) d \mu_{x, y}(\eta) \\
=\int_{-\infty}^{\infty} e^{i \eta} e^{\omega|t|}\left(\frac{2}{\pi} \int_{0}^{\infty} \frac{\cos \omega t \xi}{1+\xi^{2}} d \xi\right) d \mu_{x, y}(\eta) \\
=\int_{-\infty}^{\infty} e^{i \eta t} d \mu_{x, y}(\eta) .
\end{aligned}
$$


From these identities we conclude that the expression

$$
\int_{-\infty}^{\infty} e^{\omega|t|+i \xi t} \cdot 2 \omega\left(\left(\omega^{2} I+(\xi I-A)^{2}\right)^{-1} x, y\right) d \xi
$$

does not depend on the choice of $\omega$. So for each $t$ in $\mathbf{R}$ there exists a continuous linear map $P_{t}: \mathbf{H} \rightarrow \mathbf{H}$ such that

$$
\begin{aligned}
\left(P_{t} x, y\right) & =(2 \pi)^{-1} \int_{-\infty}^{\infty} e^{\omega|t|+i \xi t} \cdot 2 \omega\left(\left(\omega^{2} I+(\xi I-A)^{2}\right)^{-1} x, y\right) d \xi \\
& =(2 \pi)^{-1} \int_{-\infty}^{\infty} e^{i \eta t} d \mu_{x, y}(\eta)
\end{aligned}
$$

Next pick $\lambda$ in $\mathbf{C}, \operatorname{Re} \lambda>0$. From (8) and (5) it follows that

$$
\begin{aligned}
\int_{0}^{\infty} e^{-\lambda t}\left(P_{t} x, y\right) d t & =\frac{1}{2 \pi} \int_{-\infty}^{\infty}(\lambda-i \eta)^{-1} d \mu_{x, y}(\eta) \\
& =\left((\lambda I-i A)^{-1} x, y\right) .
\end{aligned}
$$

Since $\sup \left\{\left|\left(P_{t} x, y\right)\right|: t \in \mathbf{R}\right\}$ is finite and since $\lim _{t \rightarrow 0}\left(P_{t} x, y\right)=$ $\left(P_{0} x, y\right)$, it follows from (9) and (7) that

$$
\left(P_{0} x, y\right)=\lim _{\lambda \rightarrow \infty} \lambda \int_{0}^{\infty} e^{-\lambda t}\left(P_{t} x, y\right) d t=(2 \pi)^{-1} \mu_{x, y}(\mathbf{R})=(x, y) .
$$

Hence $P_{0}=I$. From (9) it also follows that

$$
\int_{0}^{\infty} e^{-\lambda t} P_{t} x d t=(\lambda I-i A)^{-1} x
$$

Since the map $\lambda \mapsto(\lambda I-i A)^{-1}, \operatorname{Re} \lambda>0$, satisfies the resolvent equation, to wit

$$
\begin{aligned}
& (\lambda I-i A)^{-1}-(\mu I-i A)^{-1} \\
& \quad=(\mu-\lambda)(\lambda I-i A)^{-1}(\mu I-i A)^{-1}, \quad \operatorname{Re} \lambda, \operatorname{Re} \mu>0,
\end{aligned}
$$

it follows that $\left\{P_{s}: s \geq 0\right\}$ is a semigroup. This means

$$
P_{s+t}=P_{s} \circ P_{t}, \quad s, t \geq 0 .
$$

This semigroup is weakly continuous. By a standard result on semigroups it is strongly continuous. By (10) its generator is given by $i A$. Similarly the family $\left\{P_{-s}: s \geq 0\right\}$ is a strongly continuous semigroup with generator $-i A$. Consequently the collection $\left\{P_{s}: s \in \mathbf{R}\right\}$ is a strongly continuous group for which $\sup \left\{\left|\left(P_{s} x, y\right)\right|: s \in \mathbf{R}\right\}$ is finite for each $x, y$ in $\mathbf{H}$. It follows that $\sup \left\{\left\|P_{s}\right\|: s \in \mathbf{R}\right\}$ is finite. This proves (ii).

(ii) $\Rightarrow$ (v) Trivial. 
(v) $\Rightarrow$ (vi) Let $-i A$ be the generator of a group $\left\{P_{s}: s \in \mathbf{R}\right\}$ for which (v) is satisfied. Then $A$ is closed, $\lim _{\lambda \rightarrow \infty} \lambda\left((\lambda I+i A)^{-1} x, y\right)=(x, y), x, y$ in $\mathbf{H}$ and $(\lambda I-i A)^{-1}$ exists for $\operatorname{Re} \lambda \neq 0$. By Plancherel's theorem in $L^{2}(\mathbf{R}, \mathbf{H})$ we conclude

$$
\begin{aligned}
2 \omega \int_{-\infty}^{\infty} \mid & \left(\left(\omega^{2} I+(\xi-A)^{2}\right)^{-1} x, y\right) \mid d \xi \\
= & 2 \omega \int_{-\infty}^{\infty}\left|((\omega-i \xi) I+i A)^{-1}((\omega+i \xi) I-i A)^{-1} x, y\right| d \xi \\
= & 2 \omega \int_{-\infty}^{\infty}\left|((\omega+i \xi) I-i A)^{-1} x,\left((\omega+i \xi) I-i A^{*}\right)^{-1} y\right| d \xi \\
\leq & 2 \omega \int_{-\infty}^{\infty}\left\|((\omega+i \xi) I-i A)^{-1} x\right\|\left\|\left((\omega+i \xi) I-i A^{*}\right)^{-1} y\right\| d \xi \\
\leq & 2 \omega\left(\int_{-\infty}^{\infty}\left\|((\omega+i \xi) I-i A)^{-1} x\right\|^{2} d \xi\right)^{1 / 2} \\
& \times\left(\int_{-\infty}^{\infty}\left\|\left((\omega+i \xi) I-i A^{*}\right)^{-1} y\right\|^{2} d \xi\right)^{1 / 2} \\
= & 2 \omega\left(\int_{-\infty}^{\infty}\left\|\int_{0}^{\infty} e^{-(\omega+i \xi) s} P_{-s} x d s\right\|^{2} d \xi\right)^{1 / 2} \\
& \times\left(\int_{-\infty}^{\infty}\left\|\int_{0}^{\infty} e^{-(\omega+i \xi) s} P_{s}^{*} y d s\right\|^{2} d \xi\right)^{1 / 2} \\
= & 2 \omega \cdot 2 \pi\left(\int_{0}^{\infty} e^{-2 \omega s}\left\|P_{-s} x\right\|^{2} d s\right)^{1 / 2}\left(\int_{0}^{\infty} e^{-2 \omega s}\left\|P_{s}^{*} y\right\|^{2} d s\right)^{1 / 2} \\
\leq & 2 \pi \cdot\left(\sup _{\omega>0} 2 \omega \int_{0}^{\infty} e^{-2 \omega s}\left\|P_{-s} x\right\|^{2} d s\right)^{1 / 2} \\
& \times\left(\sup _{\omega>0} 2 \omega \int_{0}^{\infty} e^{-2 \omega s}\left\|P_{s}^{*} y\right\|^{2} d s\right)^{1 / 2} \cdot
\end{aligned}
$$

From (v) and from Lemma 1.1 it follows that the latter expression is finite. Hence

$$
\sup \left\{2 \omega \int_{-\infty}^{\infty}\left|\left(\left(\omega^{2} I+(\xi I-A)^{2}\right)^{-1} x, y\right)\right| d \xi: \omega>0\right\}
$$

is finite. Whence (vi) follows. 
REMARK. Under suitable modifications the implication (vi) $\Rightarrow$ (ii) is valid in Banach spaces too.

We conclude with an example of a group $\left(P_{s}: s \in \mathbf{R}\right)$ of linear operators acting on $L^{2}(\mathbf{R})$ which is not bounded but for which

$$
\sup \left\{(2 t)^{-1} \int_{-t}^{+t}\left\|P_{s} f\right\|^{2} d s: t>0\right\}
$$

is finite for each $f$ in $L^{2}(\mathbf{R})$.

ExAmple 2. Fix $0<2 \gamma<1$ and put $\varphi(x)=(1+|x|)^{\gamma}, x \in \mathbf{R}$. Define for each $s$ in $\mathbf{R}$ the operator $P_{s}: L^{2}(\mathbf{R}) \rightarrow L^{2}(\mathbf{R})$ by

$$
P_{s} f(x)=\frac{\varphi(x+s)}{\varphi(x)} f(x+s), \quad x \in R, f \in L^{2}(\mathbf{R}) .
$$

Then the family $\left\{P_{s}: s \in \mathbf{R}\right\}$ is a strongly continuous group for which $\left\|P_{s}\right\|=\varphi(s)$ and for which

$$
\sup \left\{(2 t)^{-1} \int_{-t}^{+t}\left\|P_{s} f\right\|^{2} d s: t>0\right\}
$$

is finite for each $f$ in $L^{2}(\mathbf{R})$. Define the operator $A$ as follows. Its domain $D(A)$ is given by

$$
D(A)=\left\{f \in L^{2}(\mathbf{R}): f^{\prime} \in L^{2}(\mathbf{R})\right\}
$$

and $A f, f \in D(A)$, is given by

$$
A f(x)=i f^{\prime}(x)+\frac{i \gamma x}{|x|} \cdot \frac{1}{1+|x|} \cdot f(x), \quad x \in \mathbf{R} .
$$

Then $-i A$ generates the group $\left\{P_{s}: s \in \mathbf{R}\right\}$. Since $\sup \left\{\left\|P_{s}\right\|: s \in \mathbf{R}\right\}=\infty$, the operator $A$ is not similar to a self-adjoint one.

ACKNOWLEDGEMENT. The author is indebted to the referee for reference [5]. He is also grateful to R. A. Hirschfeld (Antwerp) for some valuable comments. He is obliged to the National Fund for Scientific Research (NFWO) of Belgium and to the University of Antwerp (UIA) for their material support.

Added in proof. An announcement of these results can be found in: J. A. Van Casteren, Opérateurs similaires à des opérateurs unitaires, pp. 453-455 of Actualités Mathématiques: Actes du 6e Congrès du Groupement des Mathèmaticiens d'Expression Latine, Luxembourg 7-12 septembre 1981, edited by J. P. Pier and published by Gauthier-Villars, Paris 1982. 


\section{REFERENCES}

1. P. Duren, Theory of $H^{p}$ Spaces, Academic Press (New York, 1970).

2. R. E. Edwards ; and G. I. Gaudry, Littlewood-Paley and multiplier theory, Springer Verlag (Berlin, 1977).

3. H. G. Garnir, K. R. Unni and J. H. Williams (editors), Functional analysis and its applications, Lecture Notes in Math. 399, Springer Verlag (Berlin, 1974).

4. E. Hewitt and K. A. Ross, Abstract harmonic analysis I. Springer Verlag (Berlin, 1963).

5. J. G. Stampfli, A local spectral theory for operators, III: Resolvents, spectral sets and similarity, Trans. Amer. Math. Soc., 168 (1972), 133-151.

6. E. M. Stein, Singular integral operators and differentiability properties of functions, Princeton Math. Series 30 (Princeton 1970).

7. B. Sz.-Nagy, On uniformly bounded linear transformations in Hilbert Space, Acta Sci. Math., 11 (1947), 152-157.

8. B. Sz-Nagy and C. Foiaş, Analysis harmonique de opérateurs de l'espace de Hilbert, Akadémiai Kiadó (Budapest, 1967).

9e. J. A. Van Casteren, A problem of Sz.-Nagy, Acta Sci. Math., 42 (1980), 189-194.

10. K. Yosida, Functional analysis, 3rd ed., Springer Verlag (Berlin, 1971).

Received February 27, 1981 and in revised form September 14, 1981.

UNIVERSITAIRE INSTELLING ANTWERPEN

UNIVERSITEITSPLEIN 1

2610 WILRIJK, BELGIUM 



\section{PACIFIC JOURNAL OF MATHEMATICS \\ EDITORS}

DONALD BABBITT (Managing Editor)

University of California

Los Angeles, CA 90024

\section{Hugo Rossi}

University of Utah

Salt Lake City, UT 84112

C. C. Moore and Arthur Ogus

University of California

Berkeley, CA 94720
J. DugundiI

Department of Mathematics

University of Southern California

Los Angeles, CA 90089-1113

R. FINN and H. SAMELSON

Stanford University

Stanford, CA 94305

ASSOCIATE EDITORS
R. ARens
E. F. BECKENBACH
B. H. NeumanN
F. WOLF
K. YoshidA (1906-1982)

\section{SUPPORTING INSTITUTIONS}

UNIVERSITY OF ARIZONA

UNIVERSITY OF BRITISH COLUMBIA

CALIFORNIA INSTITUTE OF TECHNOLOGY

UNIVERSITY OF CALIFORNIA

MONTANA STATE UNIVERSITY

UNIVERSITY OF NEVADA, RENO

NEW MEXICO STATE UNIVERSITY

OREGON STATE UNIVERSITY
UNIVERSITY OF OREGON

UNIVERSITY OF SOUTHERN CALIFORNIA

STANFORD UNIVERSITY

UNIVERSITY OF HAWAII

UNIVERSITY OF TOKYO

UNIVERSITY OF UTAH

WASHINGTON STATE UNIVERSITY

UNIVERSITY OF WASHINGTON 


\section{Pacific Journal of Mathematics}

Vol. 104, No. 1

May, 1983

Nestor Edgardo Aguilera and Eleonor Ofelia Harboure de Aguilera, On

the search for weighted norm inequalities for the Fourier transform $\ldots \ldots .1$

Jin Akiyama, Frank Harary and Phillip Arthur Ostrand, A graph and its complement with specified properties. VI. Chromatic and achromatic numbers ......................................... 15

Bing Ren Li, The perturbation theory for linear operators of discrete type . . . 29

Peter Botta, Stephen J. Pierce and William E. Watkins, Linear

transformations that preserve the nilpotent matrices .............. 39

Frederick Ronald Cohen, Ralph Cohen, Nicholas J. Kuhn and Joseph

Alvin Neisendorfer, Bundles over configuration spaces .......... 47

Luther Bush Fuller, Trees and proto-metrizable spaces . . . . . . . . . . 55

Giovanni P. Galdi and Salvatore Rionero, On the best conditions on the

gradient of pressure for uniqueness of viscous flows in the whole space . . 77

John R. Graef, Limit circle type results for sublinear equations $\ldots \ldots \ldots \ldots 85$

Andrzej Granas, Ronald Bernard Guenther and John Walter Lee,

Topological transversality. II. Applications to the Neumann problem for

$y^{\prime \prime}=f\left(t, y, y^{\prime}\right) \ldots \ldots \ldots \ldots \ldots \ldots \ldots \ldots \ldots \ldots \ldots \ldots \ldots \ldots \ldots . \ldots 5$

Richard Howard Hudson and Kenneth S. Williams, Extensions of

theorems of Cunningham-Aigner and Hasse-Evans . . . . . . . . . . . 111

John Francis Kurtzke, Jr., Centralizers of irregular elements in reductive algebraic groups

James F. Lawrence, Lopsided sets and orthant-intersection by convex

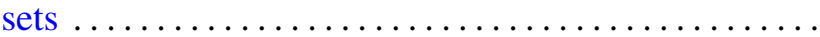

Åsvald Lima, G. H. Olsen and U. Uttersrud, Intersections of $M$-ideals and

$G$-spaces

Wallace Smith Martindale, III and C. Robert Miers, On the iterates of derivations of prime rings

Thomas H. Pate, Jr, A characterization of a Neuberger type iteration procedure that leads to solutions of classical boundary value problems

Carl L. Prather and Ken Shaw, Zeros of successive iterates of multiplier-sequence operators

Billy E. Rhoades, The fine spectra for weighted mean operators

Rudolf J. Taschner, A general version of van der Corput's difference theorem

Johannes A. Van Casteren, Operators similar to unitary or selfadjoint ones 